\title{
Regional Brain Activity During Rest and Gastric Water Load in Subtypes of Functional Dyspepsia: A Preliminary Brain Functional Magnetic Resonance Imaging Study
}

\begin{abstract}
Yanwen Chen, ${ }^{1,2}$ Ruifeng Wang, ${ }^{1,3}$ Bo Hou, ${ }^{4}$ Feng Feng, ${ }^{4}$ Xiucai Fang, ${ }^{1}$ Liming Zhu, ${ }^{1}$ Xiaohong Sun, ${ }^{1}$ Zhifeng Wang, ${ }^{1}$ and Meiyun Ke ${ }^{1 *}$
${ }^{\prime}$ Department of Gastroenterology, Peking Union Medical College Hospital, Chinese Academy of Medical Sciences, Beijing; China; ${ }^{2}$ Department of Gastroenterology, Ruijin Hospital Affiliated to Shanghai Jiaotong University School of Medicine, Shanghai, China; ${ }^{3}$ Department of Gastroenterology, No.4 Hospital Affiliated to Harbin Medical University, Harbin, Heilongjiang Province, China; and ${ }^{4}$ Department of Radiology, Peking Union Medical College Hospital, Chinese Academy of Medical Sciences, Beijing; China
\end{abstract}

\section{Background/Aims}

Functional dyspepsia (FD) remains a great clinical challenge since the FD subtypes, defined by Rome III classification, still have heterogeneous pathogenesis. Previous studies have shown notable differences in visceral sensation processing in the CNS in FD compared to healthy subjects (HS). However, the role of CNS in the pathogenesis of each FD subtype has not been recognized.

\section{Methods}

Twenty-eight FD patients, including 10 epigastric pain syndrome (EPS), 9 postprandial distress syndrome (PDS), and 9 mixed-type, and $10 \mathrm{HS}$, were enrolled. All subjects underwent a proximal gastric perfusion water load test and the regional brain activities during resting state and water load test were investigated by functional magnetic resonance imaging.

\section{Results}

For regional brain activities during the resting state and water load test, each FD subtype was significantly different from $\mathrm{HS}(P<$ 0.05). Focusing on EPS and PDS, the regional brain activities of EPS were stronger than PDS in the left paracentral lobule, right inferior frontal gyrus pars opercularis, postcentral gyrus, precuneus, insula, parahippocampal gyrus, caudate nucleus, and bilateral cingulate cortices at the resting state $(P<0.05)$, and stronger than PDS in the left inferior temporal and fusiform gyri during the water load test $(P<0.05)$.

\section{Conclusions}

Compared to HS, FD subtypes had different regional brain activities at rest and during water load test, whereby the differences displayed distinct manifestations for each subtype. Compared to PDS, EPS presented more significant differences from HS at rest, suggesting that the abnormality of central visceral pain processing could be one of the main pathogenesis mechanisms for EPS.

(J Neurogastroenterol Motil 2018;24:268-279)

Key Words

Dyspepsia; Epigastric pain syndrome; Functional neuroimaging; Post-prandial distress syndrome; Regional homogeneity

Received: June 22, 2017 Revised: November 2, 2017 Accepted: November 20, 2017

(a) This is an Open Access article distributed under the terms of the Creative Commons Attribution Non-Commercial License (http://creativecommons. org/licenses/by-nc/4.0) which permits unrestricted non-commercial use, distribution, and reproduction in any medium, provided the original work is properly cited.

*Correspondence: Meiyun Ke, MD Department of Gastroenterology, Peking Union Medical College Hospital, Chinese Academy of Medical Sciences, 1 Shuaifuyuan, Wangfujing, Dongcheng District, Beijing 100730, China

Tel: +86-10-69151963, Fax: +86-10-69151963, E-mail: kemypumch2006@aliyun.com 


\section{Introduction}

Functional dyspepsia (FD), a common but heterogeneous disease, remains a great burden to the healthcare system since the mechanisms underlying the pathogenesis of FD have yet not been clearly elucidated. Multiple factors, including increased acid exposure, Helicobacter pylori infection, gastroduodenal inflammation, immune factors, dietary and life style, genetic factors and environmental factors may contribute to incidence of FD. ${ }^{1}$ It is noticeable that in clinical practice, FD patients with different major clinical manifestations tend to respond differently to the same treatment regimen. Since previous pathophysiological studies did not involve segregation of the FD subjects into different subtypes of FD according to the Rome III/IV criteria, there was no proper comparison between the mechanisms underlying the different pathogenesis of the heterogeneous FD subtypes. Visceral hypersensitivity is nevertheless one of the most important mechanisms underlying the incidence of various functional gastrointestinal disorders such as $\mathrm{FD}{ }^{2}$ In a study by Tack et al, ${ }^{3} \mathrm{FD}$ patients with visceral hypersensitivity had significantly higher prevalence of epigastric pain symptoms than those with normal visceral sensitivity, indicating that visceral hypersensitivity may be more closely related to the pathogenesis of epigastric pain syndrome (EPS) than other FD subtypes. The CNS is the center of visceral sensation processing and therefore, using brain imaging studies to monitor and compare the variations in regional brain activities of healthy subjects (HS) and FD patients can enable us to understand the role of visceral hypersensitivity in FD pathogenesis. So far, $\mathrm{H}_{2}{ }^{15} \mathrm{O}$-positron emission topography (PET) and blood-oxygen-level-dependent (BOLD) functional magnetic resonance imaging (fMRI) have been frequently used in functional brain imaging studies. Several studies have demonstrated that FD patients have differences in activities of brain regions related to visceral pain when compared to HS. ${ }^{4-6}$ However, the subjects were not further stratified according to Rome III/IV criteria in previous studies, therefore the impact of the pathophysiological heterogeneity within FD on final results could not be clearly elucidated.

This study aims to observe the differences of regional brain activities among all FD subtypes and compare them to HS, whereby fMRI would be used to monitor and observe the variations in brain activity when all the subjects would be in the resting state (no stimulation) and when they are exposed to proximal gastric water load stimulation.

\section{Materials and Methods}

\section{Subjects}

Consecutive patients aged between 20 and 65 years-old and right-handed, who met the Rome III diagnostic criteria for FD at the outpatient gastroenterology clinics of Peking Union Medical College Hospital were investigated. The patients with moderate to severe symptoms and who were voluntarily willing to participate in the study were enrolled.

Patients were excluded if they: (1) had abnormal findings in the complete blood count, fecal routine, occult blood, liver and renal functions tests, electrolytes, and abdominal ultrasound during the recent 3 months with clinical significance; (2) suffered from other chronic diseases which could also induce dyspepsia symptoms (including diabetes mellitus, peptic ulcer, chronic liver disease, gastrointestinal and hepatic malignancy, parasite infection, chronic pancreatic disease, chronic renal failure, hyperthyroidism, hypothyroidism, disturbance of water and electrolytes, and chronic exhausting disease); (3) were given long-term medications which could influence and compromise gastrointestinal functions, including calcium channel blockers, non-steroid anti-inflammatory drugs; (4) had previous medical history of abdominal or pelvic surgery; (5) had heavy smoking history (more than 10 cigarettes per day) or heavy alcohol consumption history (more than 75 gram of alcohol per day) within the recent year; (6) had been diagnosed with any psychiatric disease; and (7) had any contraindication for MRI investigation.

Simultaneously, HS were recruited for this study. The inclusion criteria for HS were: (1) aged between 20 and 65 years old; (2) no previous episode of dyspepsia symptoms in the recent year; and (3) right-handed. Volunteers were excluded if they: (1) had abnormal laboratory findings in the recent 3 months with clinical significance; (2) had a hospitalization history during the recent half year; (3) have chronic disease history; (4) had heavy smoking or alcohol consumption history, psychiatric disease history, or contraindications for MRI study — same as items (5)-(7) of exclusion criteria for FD patients.

All subjects signed an informed consent before inclusion in the study. This study was approved by the Ethics Committee of the Peking Union Medical College Hospital (IRB No. S-199). 


\section{Questionnaire Evaluation}

The questionnaire involved a series of basic information of the subjects including age, gender, height, weight, nationality, occupation, academic qualifications, marital status, financial background, social satisfaction, and proportion of physical labor/activity in daily routine. As far as the evaluation of the symptoms is concerned, with respect to the Rome III criteria for the diagnosis of digestive symptoms, the frequency and level of 8 symptoms were evaluated as shown below: postprandial fullness discomfort, early satiety, upper abdominal pain, upper abdominal flatulence, epigastric burning sensation, nausea, vomiting, and belching. The frequency of these symptoms was quantitated as follows: a score of 0 for no incidence at all, or incidence $<1$ day per month, 1 day per month or 2-3 days per month; a score of 1 was attributed for symptoms manifesting for 1-2 days per week; a score of 2 was attributed for symptoms manifesting for 3-4 days per week and a score of 3 for symptoms manifesting over 5-7 days per week. The level of symptoms was evaluated as follows: 0 , none; 1 , mild: the symptom can only be felt when extra attention is paid; 2 , moderate: the symptom is obvious and affects part of patients' work and life; 3, severe: the symptom is serious and unbearable which significantly affects patients' work and life; and the options 0 to 3 were attributed a score of $0,1,2$, and 3 respectively. The product of the severity and frequency scores of each symptom was added together and hence, the dyspepsia symptom scoring was calculated in order to rule out patients merely with mild symptom.

\section{Magnetic Resonance Imaging Acquisition}

The $\mathrm{fMRI}$ examination was performed for all subjects from 6 PM to 8 PM (Fig. 1). Prior to the examination, the subjects were required to fast for 6 to 8 hours. Before the examination, a nasogastric tube (Baitong LINK-01-2; Beijing L\&Z Medical Technology Development Co. Ltd, Beijing, China) was intubated via the nasal cavity down to $10 \mathrm{~cm}$ below the esophagogastric junction and then the subjects were asked to rest in the supine position for 10 minutes. Structural images were acquired during the first 15 minutes to rule out any anatomic abnormality. BOLD images of the subject at rest and under task state (water load test) were taken. During the task state, pure water of $37^{\circ} \mathrm{C}$ was perfused simultaneously at the speed of $300 \mathrm{~mL} / \mathrm{min}$ for 4 minutes through the gastric tube connected with a gravity drip tube (Baitong LINK-01-2;). Every subject was ordered to elevate the right leg as a signal to stop perfusion when he/she felt unbearable epigastric bloating or pain.

The MRI device used was the GE Signa VH/i Excite II 3.0T MRI system (GE Healthcare, Waukesha, WI, USA) with an 8-channel phase-arrayed head coil. In order to maintain consistency, the whole scanning procedure was conducted by the same

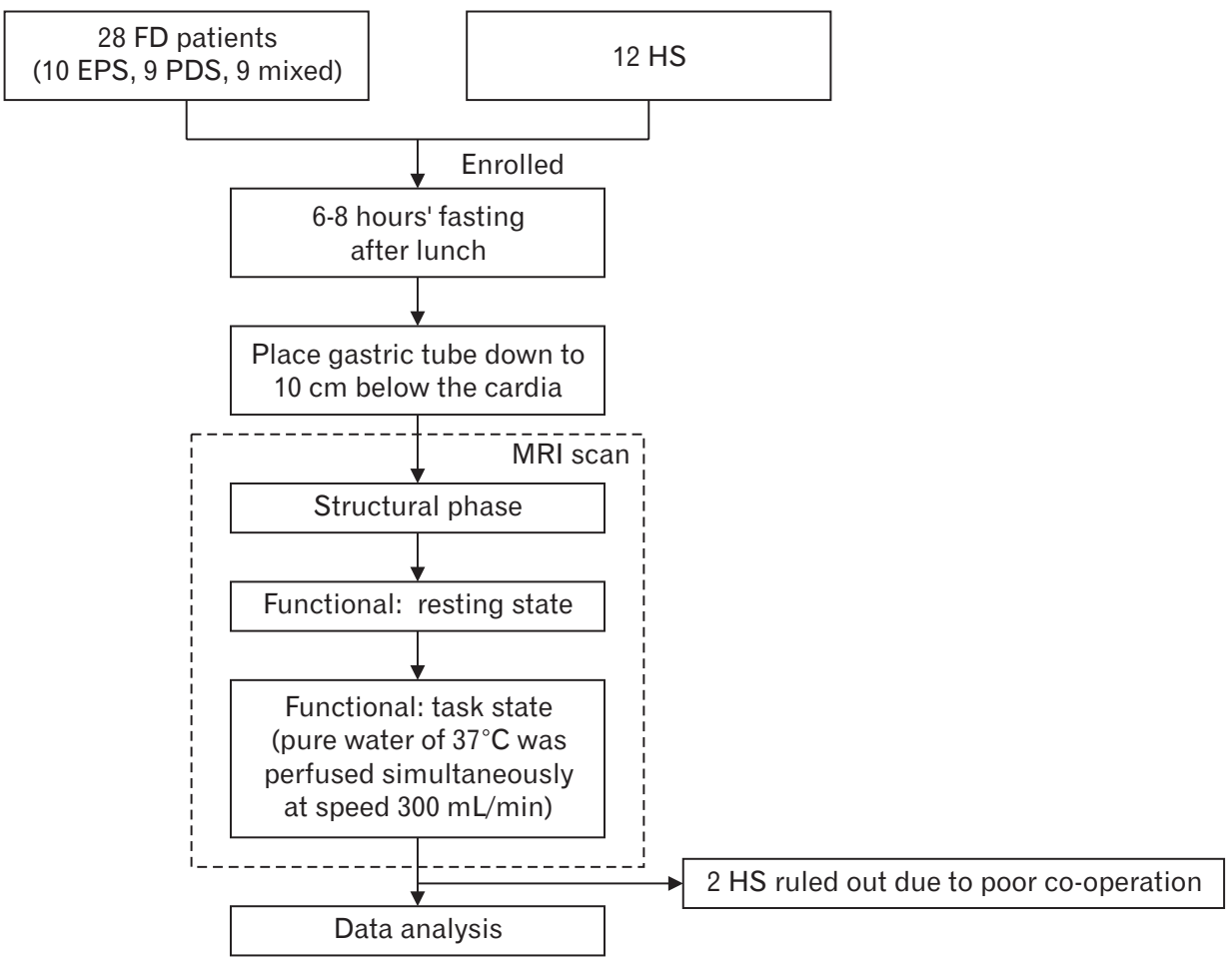

Figure 1. The flowchart diagram showing how this study was performed on the participants. FD, functional dyspepsia; EPS, epigastric pain syndrome; PDS, postprandial distress syndrome; HS, healthy subjects. 
experienced operator. Structural MRI sequences included T1WI, T2WI, and T2-FLAIR. T1 weighted whole brain volume images were acquired using the 3-dimensional fast spoiled gradient echo (3DFSPGR) sequence afterwards. BOLD imaging used the gradient echo-based echo planar imaging sequence (GRE-EPI), with the scanning parameters as follows: echo time 23 milliseconds, repetition time 2000 milliseconds, flip angle $90^{\circ}$, number of exciation 1.00 , field of view $24 \mathrm{~cm} \times 24 \mathrm{~cm}$, imaging matrix $64 \times 64$, and the same setting of slice thickness and slice gap as the axial T1WI scans. All images were reviewed to guarantee imaging quality.

\section{Statistical Methods}

The software SPSS version 17.0 (IBM Corp, Beijing, China) was used for statistical analysis. For quantitative data, the nonparametric Kruskal-Wallis method was applied for group compari-

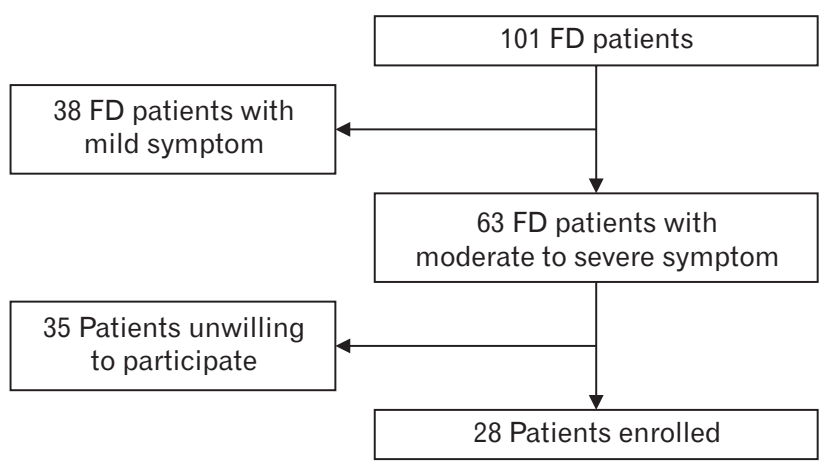

Figure 2. Screening and exclusion chart for the patient selection. FD, functional dyspepsia. son (small sample size), and the minimum and maximum values of each group were tabulated. For enumeration data, Pearson $\chi^{2}$ test was used, while Fisher exact test was applied if more than one-fifth of the cells valued less than 5 .

Analysis of fMRI data involved the following 3 steps: (1) preprocessing: the software DPARSF based on SPM8 (http://www. fil.ion.ucl.ac.uk/spm/) was applied to image pre-processing in the Matlab (MathWorks Inc, Natick, MA, USA) R2010b environment. ${ }^{7}$ Functional images were finally converted to images in accordance with the Montréal Neurological Institute standard brain coordinate system. During this step, any subject with head displacement exceeding $3 \mathrm{~mm}$ or tilt exceeding $3^{\circ}$ was ruled out. (2) Calculating regional homogeneity (ReHo) image: $\mathrm{ReHo}$ is a method using Kendall's coefficient concordance to measure the similarity of the time series of a given voxel to those of its nearest neighbors in a voxel-wise way, ${ }^{8}$ which could be used to compare differences of regional brain activities during various statuses. Respective ReHo images of every subject during resting or task state were obtained. (3) Statistical comparisons: the software resting state fMRI data analysis toolkit (REST) was used to compare ReHo images of different groups during different states. ${ }^{9}$ Using this toolkit, image data could be statistically compared just like one-dimensional data. F value parametric diagram was calculated out based on the variance analysis of the resting state data (or the water perfusion task data) of the four groups, ie, HS, EPS, postprandial distress syndrome (PDS), and mixed type. The voxels with $P$-value less than 0.05 in the $F$ value parametric diagram were then set as regions of interest (ROI) in the comparisons among groups of the resting state data (or

Table 1. Demographic Data

\begin{tabular}{|c|c|c|c|c|c|}
\hline & $\mathrm{HS}^{\mathrm{a}}$ & EPS & PDS & Mixed type & $P$-value \\
\hline Sex ratio $(M: F)^{b}$ & $5: 5$ & $3: 7$ & $5: 4$ & $3: 6$ & 0.688 \\
\hline $\operatorname{Age}^{c}$ & $42.3(24-52)$ & $35.6(21-62)$ & $44.0(21-51)$ & $37.6(20-65)$ & 0.590 \\
\hline $\operatorname{BMI}\left(\mathrm{kg} / \mathrm{m}^{2}\right)^{\mathrm{c}}$ & - & $21.43(18.56-23.44)$ & $21.72(16.84-25.71)$ & $21.09(16.84-28.65)$ & 0.784 \\
\hline Physical labor intensity ${ }^{\mathrm{d}}$ & - & $6: 4: 0$ & $7: 2: 0$ & $8: 1: 0$ & 0.427 \\
\hline Academic qualifications & - & $0: 2: 7: 1$ & $0: 0: 4: 5$ & $0: 2: 4: 3$ & 0.208 \\
\hline Marital status ${ }^{\mathrm{f}}$ & - & $1: 9: 0: 0: 0$ & $1: 8: 0: 0: 0$ & $1: 8: 0: 0: 0$ & 1.000 \\
\hline Financial status ${ }^{g}$ & - & $1: 4: 5$ & $3: 5: 1$ & $3: 6: 0$ & 0.117 \\
\hline Social satisfaction $^{\mathrm{h}}$ & - & $1: 6: 3$ & $3: 5: 1$ & $4: 5: 0$ & 0.319 \\
\hline
\end{tabular}

${ }^{a}$ Demographic data of healthy subjects (HS) only included gender and age.

${ }^{\mathrm{b}}$ Male to female subjects ratio.

${ }^{c}$ Age and body mass index (BMI) represented as the average, minimum, and maximum values.

${ }^{\mathrm{d}}$ Numbers segmented by sign of ratio represent the number of subjects with mild, moderate, and severe physical labor intensity

${ }^{\mathrm{e}}$ Academic qualifications represented as illiteracy, primary school, high school, university, or higher.

${ }^{\mathrm{f}}$ Marital status as unmarried, married, divorced, widowed, and separated.

${ }^{g}$ Financial status represented by easing, ordinary, and straitened family economic conditions.

${ }^{\mathrm{h}}$ Social satisfaction as very satisfied, satisfied, and unsatisfied.

EPS, epigastric pain syndrome; PDS, postprandial distress syndrome; M, male; F, female. 
Table 2. Summary of Regional Brain Activities of the Whole Brain Contrasting Functional Dyspepsia Subtypes and Healthy Subjects (Resting State and Water Load Test)

\begin{tabular}{|c|c|c|c|c|c|c|c|c|}
\hline \multirow{2}{*}{ Brain lobe } & \multirow{2}{*}{ Brain region ${ }^{a}$} & & \multicolumn{3}{|c|}{ Resting state } & \multicolumn{3}{|c|}{ Water load test } \\
\hline & & & EPS vs HS & PDS vs HS & MIX vs HS & EPS vs HS & PDS vs HS & MIX vs HS \\
\hline \multirow[t]{8}{*}{ Frontal lobe } & Sup frontal gyrus & $\mathrm{L}$ & $\uparrow \downarrow$ & $\uparrow \downarrow$ & $\uparrow \downarrow$ & & & \\
\hline & $(4,6)$ & $\mathrm{R}$ & $\uparrow$ & $\uparrow$ & $\uparrow$ & & & \\
\hline & Mid frontal gyrus & $\mathrm{L}$ & $\downarrow$ & & & & & \\
\hline & $(9,10)$ & $\mathrm{R}$ & & & & & & \\
\hline & Inf frontal gyrus & $\mathrm{L}$ & $\downarrow$ & $\downarrow$ & $\downarrow$ & & & \\
\hline & $(11,44,45,47)$ & $\mathrm{R}$ & $\uparrow$ & & & & & \\
\hline & Precentral gyrus & $\mathrm{L}$ & $\uparrow$ & $\uparrow$ & $\uparrow$ & & & \\
\hline & & $\mathrm{R}$ & $\uparrow$ & $\uparrow$ & $\uparrow$ & & & \\
\hline \multirow[t]{14}{*}{ Parietal lobe } & Postcentral gyrus & $\mathrm{L}$ & & $\uparrow$ & $\uparrow$ & & & \\
\hline & $(1,3 \& 2)$ & $\mathrm{R}$ & & $\uparrow$ & $\uparrow \downarrow$ & $\uparrow$ & & \\
\hline & Paracentral lobule & $\mathrm{L}$ & $\uparrow$ & $\uparrow$ & $\uparrow$ & & & \\
\hline & $(5,1,3 \& 2)$ & $\mathrm{R}$ & $\uparrow$ & $\uparrow$ & $\uparrow$ & & & \\
\hline & Sup parietal lobule & $\mathrm{L}$ & & $\uparrow$ & $\uparrow$ & & & \\
\hline & $(5,7)$ & $\mathrm{R}$ & & & & $\uparrow$ & $\uparrow$ & \\
\hline & --Precuneus & $\mathrm{L}$ & & $\uparrow$ & $\uparrow$ & $\downarrow$ & & \\
\hline & (7) & $\mathrm{R}$ & & & & $\uparrow$ & $\uparrow$ & \\
\hline & Inf parietal lobule & $\mathrm{L}$ & & & & & & \\
\hline & & $\mathrm{R}$ & & & $\downarrow$ & & & \\
\hline & --Angular gyrus & $\mathrm{L}$ & & & & & & \\
\hline & $(40)$ & $\mathrm{R}$ & & & $\downarrow$ & & & \\
\hline & --Supramarginal & $\mathrm{L}$ & & & & $\uparrow$ & & \\
\hline & $(39)$ & $\mathrm{R}$ & & & & & & \\
\hline \multirow[t]{14}{*}{ Temporal lobe } & Sup temporal gyrus & $\mathrm{L}$ & $\downarrow$ & & & & & \\
\hline & $(38)$ & $\mathrm{R}$ & & & $\uparrow$ & & & \\
\hline & --Temporal pole - sup temporal gyrus & $\mathrm{L}$ & & & & & & \\
\hline & $(38)$ & $\mathrm{R}$ & & & $\uparrow$ & & & \\
\hline & Mid temporal gyrus & $\mathrm{L}$ & $\downarrow$ & $\uparrow$ & & $\uparrow \downarrow$ & $\uparrow \downarrow$ & $\downarrow$ \\
\hline & $(21)$ & $\mathrm{R}$ & & & & & & \\
\hline & --Temporal pole - mid temporal gyrus & $\mathrm{L}$ & $\downarrow$ & & & & & \\
\hline & $(38)$ & $\mathrm{R}$ & & & & & & \\
\hline & Inf temporal gyrus & $\mathrm{L}$ & $\downarrow$ & & & $\uparrow \downarrow$ & $\downarrow$ & $\downarrow$ \\
\hline & (20) & $\mathrm{R}$ & & & & & & \\
\hline & Fusiform gyrus & $\mathrm{L}$ & & & & $\uparrow$ & & \\
\hline & $(37)$ & $\mathrm{R}$ & & & $\downarrow$ & $\uparrow$ & $\uparrow$ & $\uparrow$ \\
\hline & Medial temporal & $\mathrm{L}$ & & & & & & \\
\hline & $(28)$ & $\mathrm{R}$ & & & $\uparrow$ & & & \\
\hline \multirow[t]{6}{*}{ Occipital lobe } & Sup occipital gyrus & $\mathrm{L}$ & $\downarrow$ & & & & $\uparrow$ & \\
\hline & & $\mathrm{R}$ & & & & & & \\
\hline & Mid occipital gyrus & $\mathrm{L}$ & $\downarrow$ & & & $\uparrow$ & $\uparrow$ & \\
\hline & & $\mathrm{R}$ & $\downarrow$ & & $\downarrow$ & & $\uparrow$ & $\uparrow$ \\
\hline & Inf occipital gyrus & $\mathrm{L}$ & & & & & & \\
\hline & & $\mathrm{R}$ & $\downarrow$ & & $\downarrow$ & $\uparrow$ & $\uparrow$ & $\uparrow$ \\
\hline
\end{tabular}


task state data). The ReHo images of EPS, PDS, and mixed type groups were respectively compared with those of HS for independent sample $t$ test (two tailed), followed with AlphaSim correction ( $P<0.05$, cluster size $\geq 54$ voxels, $\mathrm{rmm}=4$; http://afni.nimh. nih.gov/afni/doc/manual/AlphaSim). Data analysis was carried out separately for resting state and task state data.

\section{Results}

\section{Demographic and Questionnaire Evaluation Data}

Twenty-eight FD patients, including 10 cases of EPS, 9 PDS, and 9 mixed type, were enrolled in this study. Twelve HS were recruited, and finally $10 \mathrm{HS}$ were enrolled in this study.

Figure 2 shows the screening and exclusion procedure for the

Table 2. Continued

\begin{tabular}{|c|c|c|c|c|c|c|c|c|}
\hline \multirow{2}{*}{ Brain lobe } & \multirow{2}{*}{ Brain region $^{\mathrm{a}}$} & & \multicolumn{3}{|c|}{ Resting state } & \multicolumn{3}{|c|}{ Water load test } \\
\hline & & & EPS vs HS & PDS vs HS & MIX vs HS & EPS vs HS & PDS vs HS & MIX vs HS \\
\hline \multirow[t]{4}{*}{ Occipital lobe } & Cuneus & $\mathrm{L}$ & $\downarrow$ & & & & & \\
\hline & & $\mathrm{R}$ & $\downarrow$ & & $\downarrow$ & & & \\
\hline & Lingual gyrus & $\mathrm{L}$ & & & & & & \\
\hline & & $\mathrm{R}$ & & & $\downarrow$ & $\uparrow$ & $\uparrow$ & $\uparrow$ \\
\hline \multirow[t]{2}{*}{ Insula } & Insular cortex & $\mathrm{L}$ & $\downarrow$ & $\downarrow$ & $\downarrow$ & & & \\
\hline & $(13 \& 14)$ & $\mathrm{R}$ & $\uparrow$ & & & & & \\
\hline \multirow[t]{10}{*}{ Limbic system } & Amygdala & $\mathrm{L}$ & & & & & & \\
\hline & & $\mathrm{R}$ & & & & & & \\
\hline & Ant cingulate cortex & $\mathrm{L}$ & $\downarrow$ & $\downarrow$ & $\downarrow$ & & & \\
\hline & $(32)$ & $\mathrm{R}$ & & & & & & \\
\hline & Mid cingulate cortex & $\mathrm{L}$ & & & & $\downarrow$ & & \\
\hline & & $\mathrm{R}$ & & & & & & \\
\hline & Post cingulate cortex & $\mathrm{L}$ & & & & & & \\
\hline & $(23,31)$ & $\mathrm{R}$ & & & & & & \\
\hline & Parahippocampal gyrus & $\mathrm{L}$ & & & & $\uparrow$ & & \\
\hline & & $\mathrm{R}$ & $\uparrow$ & & $\uparrow$ & & & \\
\hline \multirow[t]{6}{*}{ Ganglia } & Caudate & $\mathrm{L}$ & $\downarrow(19)^{\mathrm{b}}$ & $\downarrow$ & & & & \\
\hline & & $\mathrm{R}$ & & $\downarrow$ & & & & \\
\hline & Claustrum & $\mathrm{L}$ & & & & & & \\
\hline & & $\mathrm{R}$ & & & & & & \\
\hline & Lentiform (Putamen and pallidum) & $\mathrm{L}$ & $\downarrow(7)^{\mathrm{b}}$ & $\downarrow(11)^{\mathrm{b}}$ & & & & \\
\hline & & $\mathrm{R}$ & $\uparrow(10)^{\mathrm{b}}$ & & & & & \\
\hline \multirow[t]{2}{*}{ Diencephalon } & Thalamus & $\mathrm{L}$ & $\uparrow(5)^{\mathrm{b}}$ & & & & & \\
\hline & & $\mathrm{R}$ & $\uparrow(11)^{\mathrm{b}}$ & & & & & \\
\hline \multirow[t]{2}{*}{ Brain stem } & Midbrain & & & & & & & \\
\hline & Pons & & $\downarrow$ & $\downarrow$ & & & & $\downarrow$ \\
\hline
\end{tabular}

${ }^{a}$ Numbers in parenthesis following the brain region name represent the Brodmann areas belonging to this region. Only Brodmann areas with difference are listed in this table.

${ }^{b}$ Due to the small size of ganglia and thalamus themselves, brain regions with difference smaller than 10 voxel or less than $10 \%$ of the located cluster are listed as well, with the actual voxel size shown in the following parenthesis.

EPS, epigastric pain syndrome; PDS, postprandial distress syndrome; MIX, mixed type; HS, healthy subjects; L, left; R, right.

Only the brain regions with a difference larger than 20 voxel or larger than $10 \%$ of the located cluster are listed in this table. The up arrow $\uparrow$ indicates that the former group has stronger regional brain activity than the latter group $(P<0.05)$, and the down arrow $\downarrow$ indicates the opposite $(P<$ 0.05). The up and down arrows $(\uparrow \downarrow)$ together in some brain regions indicate that in several voxels of this region the former group has stronger regional brain activity than the latter $(P<0.05)$, whereas in the other voxels of this region the former has weaker activity than the latter $(P<0.05)$. 
patient selection. No significant statistical difference was found among the four groups for sex ratio and age. There is no significant statistical difference for the body mass index, physical labor intensity, academic qualifications, marriage status, financial status and social satisfaction among EPS, PDS, and mixed type (Table 1).

\section{Differences of Regional Brain Activities Between} Functional Dyspepsia Subtypes and Healthy Subjects

Comparing EPS, PDS, or mixed type group with HS, whether during the resting or task state, we were able to monitor brain areas manifesting different brain activities. Table 2 is the summarization of these areas.

Comparisons of regional brain activities at the resting state revealed that all FD subtypes were significantly different from $\mathrm{HS}$ in the bilateral superior frontal gyri, left inferior frontal gyrus, bilateral precentral gyri, bilateral paracentral lobules, left insular cortex, and left anterior cingulate cortex $(P<0.05)$. There were other areas where the regional brain activities of each FD subtype differed from HS in variety of ways, including right insular cortex, right parahippocampal gyrus and many other regions, which can be seen in Table 2. Compared to HS, the regional brain activity of EPS at resting state was higher in right insula, right middle cingulate cortex, right parahippocampal gyrus and right prefrontal cortex, while it was lesser in the left orbitofrontal cortex, left insula, anterior cingulate cortex as shown in Figure 3A. However, the difference in the regional brain activity at the resting state between PDS and HS was located in distinct regions or exhibited different activation intensity as shown in Figure 3B.

For regional brain activities during the water load test, every FD subtype was significantly different from HS in the left middle temporal gyrus, left inferior temporal gyrus, right fusiform gyrus, right inferior occipital gyrus and right lingual gyrus $(P<0.05)$.
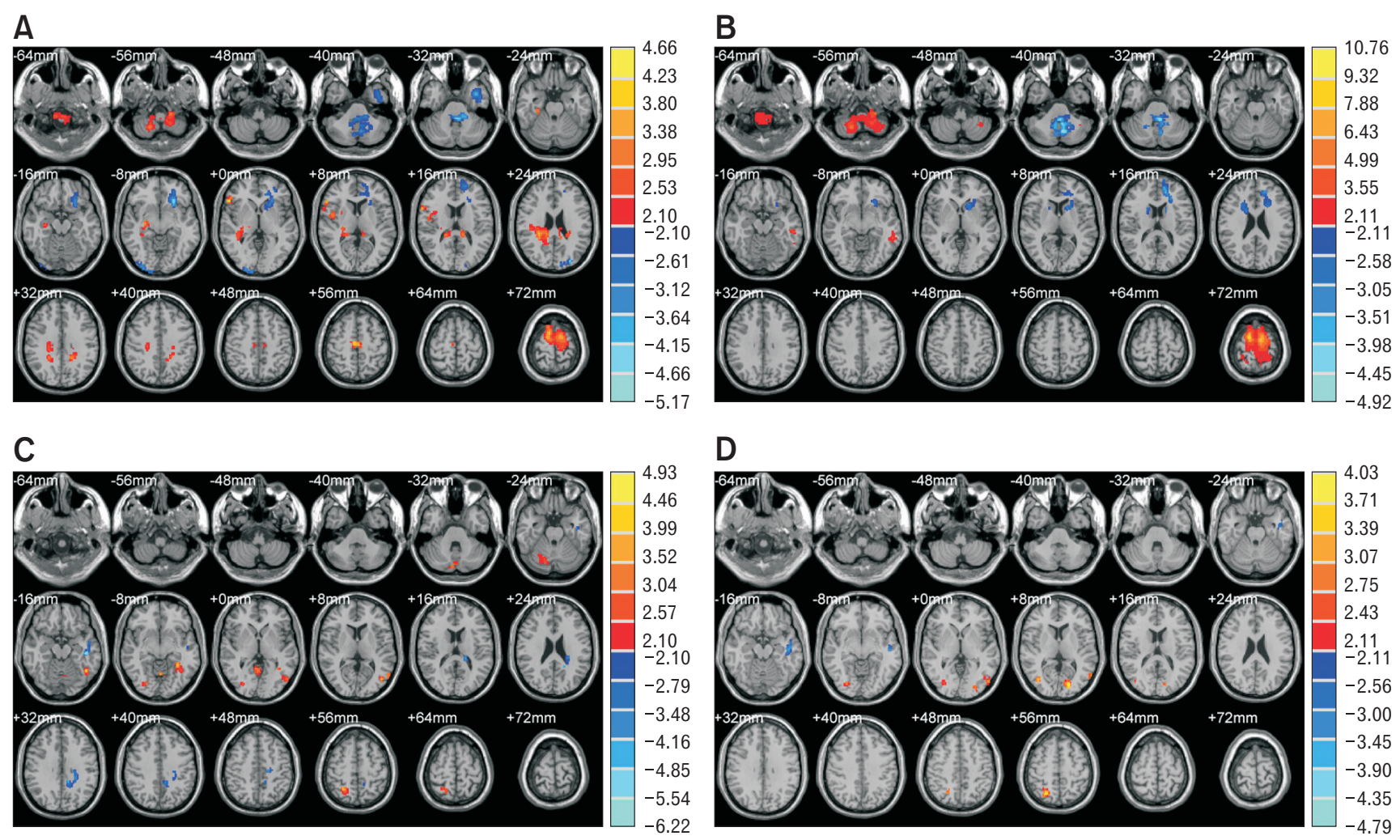

Figure 3. Regional brain activity differences between epigastric pain syndrome (EPS) (or postprandial distress syndrome [PDS]) and healthy subjects (HS) during resting state (or water load test). (A) EPS vs HS (resting state). (B) PDS vs HS (resting state). (C) EPS vs HS (water load). (D) PDS vs HS (water load). Every figure is the parameter distribution map of the $T$ value corresponding to every voxel plotted in the standard brain, after the independent sample $t$ test between two groups. The color temperature represents the magnitude of $T$ value, eg, warm colors represent the positive $T$ value, meaning the former group has stronger signal intensity than the latter group, and cold colors represent the negative $T$ value, meaning the opposite relationship between the 2 groups. The brighter the color, the greater the absolute value of $T$ value, meaning more apparent difference between the 2 groups. The color band on the right indicates the correlation between the color and the $T$ value. The layer interval in the figures is $8 \mathrm{~mm}$. 
Table 3. Regional Brain Activity Difference Between Epigastric Pain Syndrome and Postprandial Distress Syndrome During Resting State and Water Load Test

\begin{tabular}{|c|c|c|c|c|c|c|c|c|c|}
\hline \multirow{2}{*}{$\begin{array}{c}\text { Cluster } \\
\text { No. }\end{array}$} & \multirow{2}{*}{$\begin{array}{c}\text { Voxel } \\
\text { size }\end{array}$} & \multicolumn{3}{|c|}{ Peak value coordinates/mm } & \multirow{2}{*}{$\begin{array}{c}\text { Peak } T \\
\text { value }\end{array}$} & \multirow{2}{*}{ Location of peak value } & \multicolumn{3}{|c|}{ Regions included in the cluster } \\
\hline & & $\mathrm{x}$ & $\mathrm{y}$ & z & & & \multicolumn{2}{|l|}{ Structure } & Voxel size \\
\hline \multicolumn{10}{|c|}{ Resting state: EPS > PDS } \\
\hline \multirow[t]{5}{*}{1} & 861 & 27 & -30 & 21 & 3.816 & R. Cerebral white matter & R. Cingulate & & 95 \\
\hline & & & & & & & R. Caudate & & 45 \\
\hline & & & & & & & R. Hippocampus & & 27 \\
\hline & & & & & & & R. Precuneus & & 25 \\
\hline & & & & & & & R. Insula & & 20 \\
\hline 2 & 139 & -9 & -36 & 9 & 3.306 & L. Cerebral white matter & L. Cingulate & & 23 \\
\hline \multirow[t]{2}{*}{3} & 123 & 42 & -18 & 33 & 3.239 & R. Cerebral white matter & R. Postcentral gyrus & & 74 \\
\hline & & & & & & & R. Precentral gyrus & & 35 \\
\hline 4 & 54 & 39 & 12 & 30 & 3.763 & R. Inf frontal gyrus, pars opercularis & R. Inf frontal gyrus, par & rcularis & 43 \\
\hline \multirow[t]{3}{*}{5} & 72 & -6 & -24 & 57 & 3.890 & L. Paracentral lobule & L. Paracentral lobule & & 28 \\
\hline & & & & & & & BA6 (Premotor cortex) & & 28 \\
\hline & & & & & & & R. Supplementary motor & & 22 \\
\hline \multicolumn{10}{|c|}{ Resting state: EPS < PDS } \\
\hline \multirow[t]{2}{*}{6} & 61 & -36 & -54 & -51 & -2.538 & L. Cerebellum & Cerebellar tonsil & & 38 \\
\hline & & & & & & & L. Post lobe of cerebellu & & 38 \\
\hline \multirow[t]{8}{*}{7} & 388 & -39 & -12 & -9 & -4.730 & L. Sup temporal gyrus & L. Inf temporal gyrus & & 97 \\
\hline & & & & & & & L. Temporal lobe - Sup & & 82 \\
\hline & & & & & & & L. Temporal lobe - Mid & & 55 \\
\hline & & & & & & & L. BA38 (temporal pole & & 42 \\
\hline & & & & & & & L. Limbic system & & 41 \\
\hline & & & & & & & L. Uncus & & 34 \\
\hline & & & & & & & L. BA21 (mid temporal & & 32 \\
\hline & & & & & & & L. Mid temporal gyrus & & 20 \\
\hline 8 & 57 & 3 & 9 & -27 & -3.050 & undetermined & & & \\
\hline \multirow[t]{3}{*}{9} & 175 & 33 & -96 & -3 & -6.132 & R. Inf occipital gyrus (BA18) & R. Inf occipital gyrus & & 85 \\
\hline & & & & & & & R. BA18 (visual associat & tex) & 63 \\
\hline & & & & & & & R. Mid occipital gyrus & & 43 \\
\hline \multicolumn{10}{|c|}{ Water load test: EPS > PDS } \\
\hline \multirow[t]{2}{*}{1} & 69 & -45 & -54 & -12 & 6.31 & L. Inf temporal gyrus & L. Inf temporal gyrus & 24 & \\
\hline & & & & & & & L. Fusiform gyrus & 22 & \\
\hline
\end{tabular}

EPS, epigastric pain syndrome; PDS, postprandial distress syndrome; R, right; L, left; Inf, inferior; Sup, superior; Mid, middle; BA, Brodmann area. Data of this table is read from $T$ value distribution images after comparing the 2 groups during resting state or water load test. For every cluster with difference, the size with unit "voxel," peak value coordinate, peak $T$ value, location of peak value and regions included in the cluster are reported.

All clusters are corrected using AlphaSim correction, with parameters as $P<0.05$ and cluster size $\geq 54$ voxels.

The signs " $>$ " and " $<$ " represent the ReHo value of the former group stronger or weaker than the latter group respectively.

Besides, in the left middle cingulate cortex, left parahippocampal gyrus and several other regions (Table 2), each FD subtype was different from HS up to different extent $(P<0.05)$. Compared to HS, the regional brain activity of EPS during the water load test was higher in the right superior and inferior parietal lobules, right secondary somatosensory cortex, but lesser in the left middle cingulate cortex, as shown in Figure 3C. The difference of the regional brain activity during the water load test between PDS and HS was located in distinct regions or exhibited different activation intensity, as shown in Figure 3D. There were some areas, where the differ- 
ence between the brain activity of the mixed types and that of HS was more obvious than that of EPS and PDS: for example, in the right inferior parietal lobule (angular gyrus), the right fusiform gyrus, and the right lingual gyrus, the brain activity of the mixed type was lesser than that of HS, while there was no significant statistical difference among EPS, PDS, and HS; in the right superior temporal gyrus (temporal pole) and right medial temporal lobe, the brain activity of mixed types was higher than that of HS, while there was no significant statistical difference among EPS, PDS, and HS.

\section{Differences of Regional Brain Activities Between Epigastric Pain Syndrome and Postprandial Distress Syndrome Patients}

Comparing the data of EPS and PDS at resting state, the difference in the regional brain activity of the different brain areas between the 2 groups is shown in Table 3 and Figure 4. The resting brain activities of EPS were stronger than PDS in areas including right inferior frontal gyrus pars opercularis, right postcentral gyrus, left paracentral lobule, right precuneus, right insular cortex, bilateral cingulate cortices, right parahippocampal gyrus, and right caudate nucleus $(P<0.05)$. The areas with regional brain activities of EPS weaker than PDS were located in the left inferior temporal gyrus,

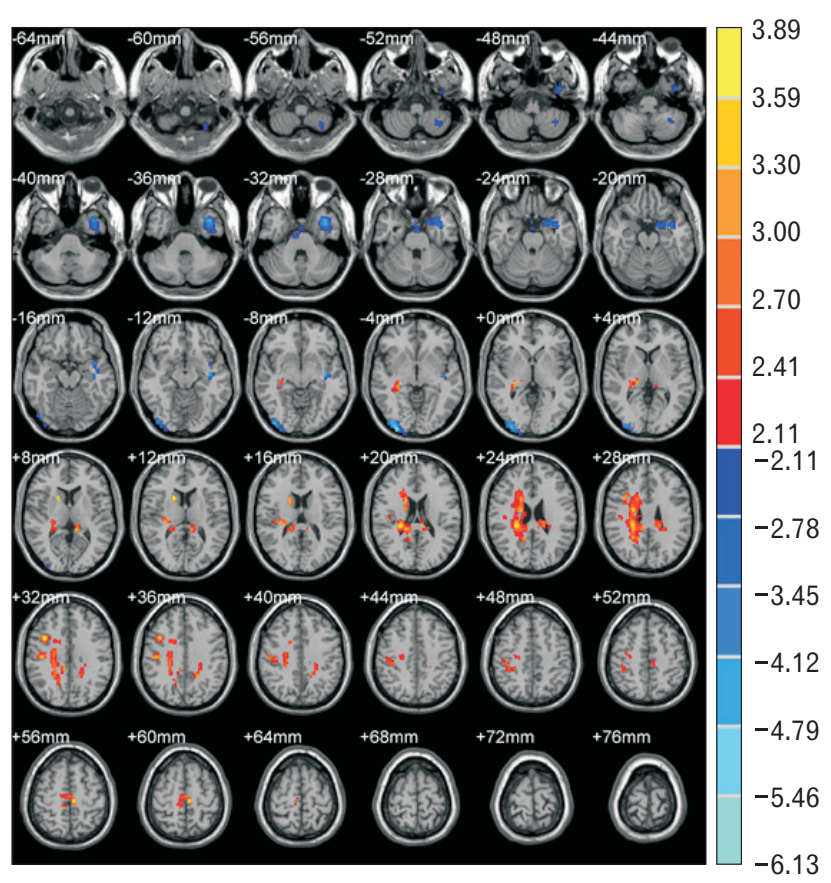

Figure 4. Regional brain activity differences between epigastric pain syndrome (EPS) and postprandial distress syndrome (PDS) during resting state. The principle of plotting is similar to Figure 3. The layer interval is $4 \mathrm{~mm}$. temporal pole, middle temporal gyrus, right middle occipital gyrus, inferior occipital gyrus, visual association cortex (Brodmann Area 18), and parts of left cerebellum $(P<0.05)$.

Statistical comparison of the task state data between EPS and PDS groups enabled us to identify the areas with different regional brain activities and is henceforth listed in Table 3. The regional brain activations of EPS during the water load test were significantly stronger than PDS in left inferior temporal gyrus and fusiform gyrus $(P<0.05)$. The areas with weaker regional brain activations of EPS than PDS did not reach statistical significance.

\section{Discussion}

This study demonstrates all FD subtypes according to the Rome III criteria differ broadly from HS in regional brain activities. Each FD subtype patients displayed different and distinct manifestation patterns at the level of regional brain activity. Among the 3 FD subtypes, EPS showed the most apparent distinction from $\mathrm{HS}$ at the resting state, while the differences of regional brain activities between EPS and PDS during the water load test were less than the resting state, which may help to explain their distinctive clinical manifestations.

Several previous neuroimaging studies have generalized the concept "visceral pain neuromatrix" as the assembly of brain regions related to visceral sensation processing, including parts of the brain stem, thalamus, insula, primary/secondary somatosensory cortex, subregions of cingulate cortex, subregions of prefrontal cortex, and parts of cerebellum. ${ }^{10}$ By exerting balloon dilation stimulation on the proximal stomach of HS ( $\mathrm{n}=15)$, Ladabaum et $\mathrm{al}^{11}$ found that the thalamus, insula, anterior cingulate cortex, caudate nucleus, periaqueductal gray matter of midbrain, cerebellum, and occipital lobe had activations under stimulation on PET scans compared to the baseline, and this was the first report about the association of the central nervous projection areas to proximal gastric stimulation. Lu et $\mathrm{al}^{12}$ used fMRI to get similar results in HS, and discovered involvement of the anterior prefrontal cortex and amygdala. The 2 studies above were consistent with the proposed concept of "visceral pain neuromatrix." Vandenberghe et al ${ }^{4}$ focused on FD patients instead and demonstrated that the distribution of brain regions activated during proximal gastric dilation in FD patients $(\mathrm{n}=13)$ with visceral hypersensitivity was similar to HS $(n=11)$, while the dilation pressure to induce activations was significantly lower in FD patients. Van Oudenhove et al ${ }^{5}$ found that the brain activities of bilateral insular cortices, left secondary and primary somatosensory cortex (SII/SI) and right dorsolateral prefrontal cortex of 
FD patients $(n=25)$ at the resting state were different from HS ( $\mathrm{n}=11)$, which indicated that FD may differ in brain functional mode independent of visceral stimulations. Based on the correlation analysis of the FD symptom scores and the signal intensity of brain regions with differences between FD $(n=40)$ and HS $(n=20)$ at the resting state, Zeng et $\mathrm{al}^{6}$ proposed that the anterior cingulate cortex, insular cortex, thalamus, middle cingulate cortex, and cerebellum may be key areas to determine the symptom severity. ${ }^{6}$

When we compared each FD subtype with HS respectively, we found that the brain regions with different activities changed with the stimulation status (resting or task state) and the patient FD subtype. This finding may indicate that the CNS is involved heterogeneously in the pathogenesis of each FD subtype. It is believed presently that some brain regions keep initiative organized activity during conscious, resting states without any tasks, forming the specific "default-mode network (DMN)," which mainly includes the medial part of the prefrontal cortex, posterior part of cingulate cortex, and bilateral inferior parietal lobes. ${ }^{13}$ Our study showed that for either PDS or mixed type FD, both the number and voxel size of clusters with different resting state activity in DMN from HS were less than EPS (Fig. 3A and 3B), indicating the central DMN abnormality may be important for EPS pathogenesis, while not so for PDS. Meanwhile we found some exceptional areas, such as the bilateral postcentral gyri, left superior parietal lobule, where the diversity between PDS and HS was stronger than that between EPS and HS, which requires deeper recognition about DMN. Comparison of regional brain activities during the water load test between EPS and PDS showed fewer areas with difference, except only several regions of left temporal lobe, indicating that volume stimulation alone, such as the $37^{\circ} \mathrm{C}$ water load, induced dyspepsia symptoms in EPS or PDS patients through central mechanisms in a similar way. The opposite findings acquired respectively from the resting state and during the water load test may explain the clinical phenomenon: both EPS and PDS have dining-associated symptoms, while only EPS patients complain of non-prandial symptoms also. However the stimulation method used as task in our study was $37^{\circ} \mathrm{C}$ water load, a physiological stimulation probably not enough to induce severe symptoms, whereas using low temperature water load $\left(\mathrm{eg}, 4^{\circ} \mathrm{C}\right)$, balloon dilation, capsaicin or other noxious stimulation mimicking daily stress factors may help to further find regional brain activity difference under stress among FD subtypes, which could be seen in another study of our team..$^{14,15}$

According to the summary of different brain activities in the resting state of all FD subtypes and HS (Table 2), we could see that in some areas every FD subtype was in the same pattern of mani- festation. For example, in the left inferior frontal gyrus (orbitofrontal cortex), left insula, left anterior cingulate gyrus (mainly the dorsal anterior cingulate gyrus), the brain activities of all FD subtypes were less than that of HS. Also, in the superior frontal gyrus and ascending frontal gyri (mainly the primary motor cortex and the premotor cortex), paracentral gyri (primary somatosensory area and somatic sensory association area), the brain activities of all FD subtypes were higher than that of HS. In other areas, the brain activity in the resting state of each FD subtype manifested differently compared to that of HS. In the left frontal gyrus (mainly the prefrontal cortex), left superior temporal gyrus, left middle temporal gyrus, left inferior temporal gyrus, left superior occipital gyrus, bilateral left middle occipital gyrus, right inferior occipital gyrus and bilateral cuneus, the brain activity of EPS was less than that of HS, which was different from the manifestation of PDS or the mixed type; In the right inferior frontal gyrus (mainly the orbitofrontal cortex), right insula, right hippocampus, right lentiform nucleus, and bilateral thalamus, the brain activity of EPS was higher than that of HS, which was different from the manifestation of PDS or the mixed type.

Recently the knowledge about visceral sensation processing has been raised from the region level to the network level, which allows comprehensive understanding. Three networks may participate in perception and processing of visceral pain, that is homeostaticafferent (including thalamus, insula, anterior part of middle cingulate cortex, and prefrontal cortex), emotion-arousal (including amygdala, subgenual part of anterior cingulate cortex, and dorsal pons) and cortical-modulation networks (including ventrolateral part of prefrontal cortex, ventromedial part of prefrontal cortex, dorsolateral part of prefrontal cortex, and subregions of parietal lobe). ${ }^{16}$ Different manifestations in these networks were found for each FD subtype in our study. We found lower resting activities of the left insula and prefrontal cortex in all FD subtypes than HS, stronger resting activities of the right insula and prefrontal cortex in EPS than HS, and no difference in these areas during the water load test. As components of homeostatic-afferent network, the insula is related to visceral perception and sensation distinguishing, ${ }^{17}$ and the prefrontal cortex takes part in integration of visceral sensation and limbic system, ${ }^{18}$ demonstrated by earlier studies. For emotionarousal network, we found lower resting activities of the anterior cingulate and dorsal pons in EPS and PDS than HS, while no difference during the water load test. Likewise, Van Oudenhove et $\mathrm{al}^{5}$ demonstrated that FD patients could not activate pregenual part of anterior cingulate cortex or deactivate dorsal pons during proximal gastric dilation stimulation, which was explained as pain regulation 
failure due to anxiety. For cortical-modulation network, our study had complicated findings between FD subtypes and HS, including that primary/secondary somatosensory cortex (SI/II) participated in activations of brain regions related to water load task. Nonetheless, previous researches did not reach the unified opinions about whether SI/II took part in visceral sensation processing. Studies of Lu et $\mathrm{al}^{12}$ and Ladabaum et al ${ }^{11}$ found that SI/II did not participate in generation of pain induced by proximal gastric dilation, while Van Oudenhove et $\mathrm{al}^{19}$ had the opposite discovery, and used subregion division with refinement. Not categorizing and defining the FD subtypes during research implementation brings the inter-group heterogeneities in which influence on the results of previous studies.

Besides the classical visceral pain neuromatrix described above, we also found FD subtypes were different from HS in some regions of the temporal lobe, occipital lobe and basal ganglia. Although the roles of these areas are not acknowledged in visceral pain processing, what we would learn could change. For instance, temporal pole (Brodmann area 38) could associate highly processed cognition information and visceral emotion reactions. ${ }^{20}$ Basal ganglia, with the key role in motion processing, could be involved in sensation integration and behavior coordination relying on its abundant neural connections. ${ }^{21}$ Ladabaum et $\mathrm{al}^{11}$ found activations of bilateral caudate nuclei during proximal gastric dilation stimulation. A recent animal study conducted by Wang et $\mathrm{al}^{22}$ demonstrated that the caudate nucleus had strong positive functional connectivity with the cingulate cortex and anterior limbic cortex. More studies are needed to recognize this sophisticated network further.

Functional MRI was used as the functional brain imaging technique in this study, which has advantages both in spatial and temporal resolutions compared to PET, and can accomplish the acquaintance of structural and functional phases on the same machine. $^{23}$ The method we used to induce symptoms of FD patients was the proximal gastric water load stimulation through nasogastric tube, which is closer to physiological feeding status than barostat balloon dilation stimulation. ${ }^{24}$ Task related fMRI studies were relatively common in the past, while the resting state has not been learned and valued until recent years. Knowledge about DMN has established the role of resting state studies. ${ }^{13} \mathrm{ReHo}$ is one of the main analytical methods of resting state fMRI studies. ${ }^{8}$ It has been confirmed that DMN calculated by ReHo method is consistent with other analytical methods. ${ }^{25}$

Our study is the first pathophysiological study about functional brain imaging of FD subtypes according to the Rome III criteria for FD. Previous studies of brain imaging in FD were either based on the Rome II criteria, or lacking subtype classification of FD.
The newest diagnosis criteria for FD, Rome IV criteria, is generally consistent with the Rome III criteria, with only a few minor differences. Hence, our study could be comparable to the future work based on the Rome IV criteria. However, the sample size was limited in this study which is to be improved in subsequent studies. We used the warm water perfusion-a type of physiological stimulationas the experimental task. In the future, varieties of non-physiological stimulations, such as cold water, capsaicin and acidic perfusion, could be used to observe the regional brain activities under stress. More pathophysiological studies based on the Rome IV typing are required to illustrate the distinct essences of $\mathrm{FD}$ subtypes.

In conclusion, FD patients manifest and undergo different regional brain activities of both resting state and water load test when compared to HS. The differences in the trends in the brain activity between each FD subtype are very distinct: compared to PDS patients, EPS patients present more apparent differences from the HS during the resting state, which may indicate that the abnormality of central visceral pain processing by $\mathrm{CNS}$ is one of the main pathogenesis mechanisms for EPS.

\section{Financial support: None.}

\section{Conflicts of interest: None.}

Author contributions: Yanwen Chen, Ruifeng Wang, and Bi Hou performed the research; Yanwen Chen analyzed the data and wrote the paper; Feng Feng, Xiucai Fang, Zhifeng Wang, and Meiyun Ke gave instructions on protocol designing, research implementation, data analysis and paper writing; and Xiucai Fang, Liming Zhu, Xiaohong Sun, and Meiyun Ke contributed to patient enrollment.

\section{References}

1. Miwa H, Watari J, Fukui H, et al. Current understanding of pathogenesis of functional dyspepsia. J Gastroenterol Hepatol, 2011;26(suppl 3):53-60.

2. Van Oudenhove L, Demyttenaere K, Tack J, Aziz Q. Central nervous system involvement in functional gastrointestinal disorders. Best Pract Res Clin GastroenterolBest Pract Res Clin Gastroenterol 2004;18:663680.

3. Tack J, Caenepeel P, Fischler B, Piessevaux H, Janssens J. Symptoms associated with hypersensitivity to gastric distention in functional dyspepsia. Gastroenterology, 2001;121:526-535.

4. Vandenberghe J, Dupont P, Van Oudenhove L, et al. Regional cerebral blood flow during gastric balloon distention in functional dyspepsia. Gastroenterology 2007;132:1684-1693. 
5. Van Oudenhove L, Vandenberghe J, Dupont $\mathrm{P}$, et al. Abnormal regional brain activity duringrest and (anticipated) gastric distension in functional dyspepsia and the role of anxiety: $\mathrm{H}_{2}{ }^{15} \mathrm{O}-\mathrm{PET}$ study. Am J Gastroenterol 2010;105:913-924.

6. Zeng F, Qin W, Liang F, et al. Abnormal resting brain activity in patients with functional dyspepsia is related to symptom severity. Gastroenterology 2011;141:499-506.

7. Chao-Gan Y, Yu-Feng Z. DPARSF: A MATLAB toolbox for "Pipeline" data analysis of resting-state fMRI. Front Syst Neurosci 2010;4:13.

8. Zang Y, Jiang T, Lu Y, He Y, Tian L. Regional homogeneity approach to fMRI data analysis. Neuroimage 2004;22:394-400.

9. Song XW, Dong ZY, Long XY, et al. REST: a toolkit for resting-state functional magnetic resonance imaging data processing. PLoS One 2011;6:e25031.

10. Van Oudenhove L, Coen SJ, Aziz Q. Functional brain imaging of gastrointestinal sensation in health and disease. World J Gastroenterol 2007; 13:3438-3445.

11. Ladabaum U, Minoshima S, Hasler WL, Cross D, Chey WD, Owyang C. Gastric distention correlates with activation of multiple cortical and subcortical regions. Gastroenterology 2001;120:369-376.

12. Lu CL, Wu YT, Yeh TC, Ho LT, Hsieh JC. Neuronal correlates of gastric pain induced by fundus distension: a 3T-fMRI study. Neurogastroenterol Motill 2004;16:575-587.

13. Raichle ME, MacLeod AM, Snyder AZ, Powers WJ, Gusnard DA, Shulman GL. A default mode of brain function. Proc Natl Acad Sci USA 2001;98:676-682.

14. Wang RF, Wang ZF, Ke MY, et al. Temperature can influence gastric accommodation and sensitivity in functional dyspepsia with epigastric pain syndrome. Dig Dis Sci 2013;58:2550-2555.

15. Chen Y, Wang R, Feng F, et al. Temperature of water load can influence regional brain activations of $\mathrm{F}$ epigastric pain syndrome. J Gastroenterol Hepatol 2013;28(suppl 3):1.
16. Mayer EA, Naliboff BD, Craig AD. Neuroimaging of the brain-gut axis: from basic understanding to treatment of functional GI disorders. Gastroenterology 2006;131:1925-1942.

17. Craig AD. Pain mechanisms: labeled lines versus convergence in central processing. Annu Rev Neurosci 2003;26:1-30.

18. Cavada C, Compañny T, Tejedor J, Cruz-Rizzolo RJ, Reinoso-Suáarez F. The anatomical connections of the macaque monkey orbitofrontal cortex. A review. Cereb Cortex 2000;10:220-242.

19. Van Oudenhove L, Dupont P, Vandenberghe J, et al. The role of somatosensory cortical regions in the processing of painful gastric fundic distension: an update of brain imaging findings. Neurogastroenterol Motil 2008;20:479-487.

20. Ding SL, Van Hoesen GW, Cassell MD, Poremba A. Parcellation of human temporal polar cortex: a combined analysis of multiple cytoarchitectonic, chemoarchitectonic, and pathological markers. J Comp Neurol 2009;514:595-623.

21. Borsook D, Upadhyay J, Chudler EH, Becerra L. A key role of the basal ganglia in pain and analgesia--insights gained through human functional imaging. Mol Pain 2010;6:27.

22. Wang Z, Bradesi S, Charles JR, et al. Pang RD, Maarek JM, Mayer EA, Holschneider DP. Functional brain activation during retrieval of visceral pain-conditioned passive avoidance in the rat. Pain 2011;152:27462756.

23. Andresen V. Visceral sensitivity testing. Best Pract Res Clin Gastroenterol 2009;23:313-24.

24. Zhang J, Sun XH, Wang ZF. Perfusion nutrient load test:a new method in assessment of gastric accommodation. Chung Hua Hsiao Hua Tsa Chih 2010;30:518-521.

25. Long XY, Zuo XN, Kiviniemi V, et al. Default mode network as revealed with multiple methods for resting-state functional MRI analysis. J Neurosci Methods 2008;171:349-355. 